\title{
Durability of automotive jounce bumper
}

\begin{abstract}
This study was carried out to predict the durability of automotive car jounce bumper using Finite Element Analysis (FEA). Fatigue life correlations were taken from literatures and it was incorporated into FEA codes. The simulated results were validated with experimental work. The FEA results showed good agreement with the experiment conducted on the jounce bumper in term of loadï displacement response. In term of the durability of the component, the fatigue life predicted shows agreement at lower fatigue strains. However, the error becomes larger as the fatigue strains become higher. The differences between the predicted fatigue life and the experimental fatigue life were discussed. Finally, the predicted crack initiation side was also validated in the experiment.
\end{abstract}

